\title{
Effect of an Educational Program on Self-efficacy of Adolescents with Thalassemia Major
}

\author{
Eman Habib Sadek ${ }^{1}$, Khalid Ibrahim Elsayh ${ }^{2}$, Fathia Zaky Mohammed ${ }^{3}$, Nahed Thabet Mohamed ${ }^{4} \&$ Sahar \\ Sedky Faheem ${ }^{5}$. \\ 1. Assistant Lecturer of Pediatric Nursing, Faculty of Nursing, Beni-Suef University, Egypt. \\ 2. Professor of Medicine, Faculty of Medicine, Assiut University, Egypt. \\ 3. 4. Assistant Professor of Pediatric Nursing, Faculty of Nursing, Assiut University, Egypt. \\ 5. Assistant Professor of Pediatric Nursing, Faculty of Nursing, Beni-Suef University, Egypt.
}

\begin{abstract}
Background: Adolescents with thalassemia major face many challenges in adjusting to their illness. Self-efficacy is a highly valuable modifiable construct for adolescents' adjustment. Coping with thalassemia major is best when they demonstrate high self-efficacy. This study aimed to assess the effect of an educational program on self-efficacy of adolescents with thalassemia major. A quasi-experimental research design was used to perform this study. A convenience sampling of (80) adolescents with thalassemia major attending the Thalassemia Center at Assiut University Children Hospital was selected. Data were collected using two tools; Tools (1):- A structured Questionnaire Interview Sheet, Tool (2):- The Self-Efficacy Scale. Results revealed that most (82.5\%) of the adolescents in the study group had moderate self-efficacy after implementing the educational program to only $27.5 \%$ in the control group. The study results reported a highly statistically significant difference between the studied adolescents' knowledge level and their self-efficacy level $(\mathrm{p}=0.00)$. Conclusion: The present study highlighted the effectiveness of the educational program on enhancing self-efficacy of adolescents with thalassemia major. So, the study recommended that the health education programs must be held frequently for adolescents with thalassemia major to increase their knowledge about the disease and how to cope with it.
\end{abstract}

\section{Keywords: Adolescents- Educational Program- Self-efficacy \& Thalassemia Major.}

\section{Introduction}

Thalassemia major (TM) is the most severe form of thalassemic disease which is a chronic hereditary disease, caused by abnormalities in the synthesis of beta chains of hemoglobin. TM is transmitted to next generations as an autosomal recessive trait, which means both parents must be affected or be carriers for the disease to be transferred to the next generation (Madmoli et al., 2019).

Thalassemia is a type of inherited anemia. Its management is complex and expensive, and requires a multiple-team approach. Optimal and sufficient clinical care is demanding. Thalassemia major is the commonest chronic hemolytic anemia among adolescents worldwide. According to the Thalassemia International Federation, only about 200, 000 children with thalassemia major are alive with about 60, 000 new children are born annually with thalassemia worldwide (Al-Kherbash et al., 2018).

Control of thalassemia depends on certain preventive health services. Preventive health services vary between premarital counseling, health education about the risks of having a child with thalassemia along with screening and genetic counseling. Preventive measures are associated with numerous benefits which include decreasing the chance of having a child affected with thalassemia major and decreasing the inevitable high medical cost (Basu, 2015).

According to the social-cognitive theory, selfefficacy means the faith one has in him/herself to perform a particular behavior. As an effective factor in promoting adolescents' quality of life, enhancing self-efficacy in adolescents with thalassemia remains so important; as it is related to the capability to achieve some important goals together with their psychological well-being (Platania et al., 2017 \& Taheri et al., 2020).

Chronic nature of thalassemia causes changes in different aspects of adolescents' life, including their self-efficacy. Over the years, regular blood transfusions and effective chelation have been able to achieve longer survival rates. But these gains will be of less value if they are not accompanied with a better quality of life and an enhanced level of selfefficacy (Ankush et al., 2019).

Thalassemia major negatively affects the adolescents' life and their psychosocial well-being. Adolescents with higher levels of self-efficacy altogether with a strong belief in their ability to handle the daily tasks are more likely to perform better in terms of achieved goals. Low self-efficacy of the affected adolescents with thalassemia major is characterized by lacking the faith in their ability to achieve daily goals. 
In those circumstances, it is important to develop certain methods guided for their assistance, in order for them to achieve an improvement in the quality of their life and self-efficacy; that would also allow them to coexist in a more peaceful way with their pathological condition (Platania et al., 2017).

Thalassemia major is a chronic disease; therefore the nurse needs to build a close bond with the adolescent who would require a continued care. The nurse should consider both psychological as well as medical needs of the family. Nurses are also essential in helping the adolescent to become aware of their own condition, teaching effective techniques for self-management, preventing further complications and helping in the transition of pediatric patients to the adult team of healthcare specialists, as well as in genetic counseling (Elsayed and Abd El-Gawad, 2015 \& Gupta, 2017). Significance of the study

Thalassemia major is a major global health challenge affecting nearly 200 million children worldwide. Globally, there are about 240 million carriers of $\beta$-thalassemia. In Egypt, $\beta$-thalassemia is considered the most common monogenic disorder with a carrier rate of almost 5.3 to $9.0 \%$, representing the most common genetically determined chronic hemolytic anemia (85.1\%) (Biswas et al., 2019 \& Sahmoud et al., 2020).

Various aspects of life of the affected adolescents and their families are usually affected and adversely influence the adolescents' physical and psychological health, quality of life of the patients and their family, and accordingly their self-efficacy (Lai et al., 2017 \& Taheri et al., 2020).

The operational definition of self-efficacy was synthesized as follows.

"Self-efficacy is a strongly subjective concept, but observational and measurable. It focuses on a person's fullest utilization of his or her cognitive capability to produce a beneficial outcome through learning by personal experience and observation. It is mediated by one's level of self-confidence, self-determinism, selfresponsibility and self-control." Operational definition are informed by data from self-report surveys that assess one's level of confidence or self-efficacy to accomplish thalassemia management tasks (Rasbash et al., 2016).

\section{Aim of the study}

The aim of this study was to assess the effect of an educational program on self-efficacy of adolescents with thalassemia major.

\section{Study hypothesis:}

Adolescents with thalassemia major assigned to the educational program have higher levels of selfefficacy more than those who don't.

\section{Subjects and Method \\ Research design:}

A quasi-experimental research design was used to conduct this study.

\section{Setting:}

This study was conducted in the Thalassemia Center at Assiut University Children Hospital. The Thalassemia Center containing ten large-sized beds and

served seven governorates in Upper Egypt from ElMinia to Aswan. Annually, it receives more than 200 cases mainly for blood transfusions. The majority of cases were diagnosed with thalassemia major beside other hematological disorders as hemophilia.

\section{Subjects}

A convenience sample composed of 80 adolescents diagnosed with thalassemia major was included in the study. Adolescents were assigned randomly into two equal groups by using a coin. The researcher used a coin to assign the subjects. After flipping the coin, the adolescent was assigned to the study group if the "king face" appeared while the "writing face" was specified for the control group according to the inclusion criteria.

Adolescents in the study group were assigned to an educational program about thalassemia major beside the routine medical care, while the adolescents in the control group received the routine care only. All adolescents who were able to read and write, willing to participate in the program, and having no training in relation to the disease were recruited into the study.

Tools of data collection

Two tools were utilized to collect data pertinent to the study.

Tool (1): A structured Questionnaire Interview Sheet: It was prepared by the researcher after reviewing the related literature.

It was divided into three parts

Part I: Demographic data of the adolescent as age, gender, birth order, number of siblings, residence and educational level.

Part II: Adolescent's medical data as age at onset of the disease, duration of the disease since first symptoms, onset of chelation therapy, frequency of blood transfusion and splenectomy. Also, it included family history of the disease e.g. (parents' degree of consanguinity, family history and affected family member). - 6 -

\section{Part III: Thalassemia Major Knowledge Questionnaire; \\ The final form of the questionnaire (the modified form) composed of 17 questions including (definition, inheritance pattern, causes, symptoms, complications, causes of complications, complications of iron overload, treatment, importance of blood transfusion,}


complications of repeated blood transfusion, importance of iron chelators, frequency of oral iron chelators administration, side effects of iron chelators, complications of splenectomy, types of foods containing small amounts of iron, types of foods rich in iron and prevention of thalassemia major)

\section{Tool (2): The Self-Efficacy Scale}

The Self-Efficacy Scale was adapted from Edwards et al., (2001) It was the first instrument validated to test self-efficacy in adolescents and adults with hemoglobinopathies as sickle cell anemia and thalassemia major. The scale contained nine questions that measured self-efficacy in the target adolescents as (daily activities, overcoming feeling of sadness, managing the daily life, managing symptoms, making friendship without fear of rejection due to facial changes, dealing with frustration of having the disease, school attendance, paying attention, accomplishing goals).

\section{Scoring system}

The final form of the Thalassemia Major Knowledge Questionnaire (the modified form) composed of 17 items and the responses were modified to be scored (2) for the complete correct answer, (1) for the incomplete answer, and (0) for wrong answer or don't know response. Knowledge score was considered weak, acceptable and good when total score were less than 13 , between 13 to 24 and more than 24 respectively (Shareef $\&$ Obaid 2015).

Responses for The Self-Efficacy Scale were scored in a 5 point likert scale ranging from "not at all sure" to "very sure" and the scores ranged from 9-45. Scores 79-20, 21-32, and 33-45 indicated low self-efficacy, moderate self-efficacy and high self-efficacy, respectively (Edwards et al., 2001).

\section{Method of Data collection}

An official Permission was obtained from the chairman of Pediatric Department to collect data from the Thalassemia Center at Assiut University Children Hospital.

\section{Pilot study}

A pilot study was carried out on eight adolescents (10 $\%)$. It was conducted to ensure the clarity of the study tools and to estimate the time required for fulfilling the questionnaire sheet. Based on the results of the pilot study, necessary modifications; were done. The modification of Thalassemia Major Knowledge Questionnaire involved an exclusion of 9 questions (the questions were excluded because of either being similar to other questions or being insignificant to the aim of the study).The Self-Efficacy Scale was modified to accommodate the difference in nature between the sickle cell disease and thalassemia major. Modification included the omission of two questions revolving around the pain and an addition of two questions; one about school attendance and another one related to making friendship without fear of rejection due to facial changes. The adolescents included in the pilot study were excluded from the study sample.

\section{Validity and reliability}

The content validity of the tools of the study was estimated by five experts in Pediatrics and the Pediatric Nursing Field. The content validity index was 0.97 for tool (1) part III and 0.90 for tool (2). The internal consistency was tested for tool (2) using the Cronbach's alpha and it was $\mathrm{R}=0.83$.

\section{Ethical considerations}

Ethical approval was obtained from the Ethical Committee at the Faculty of Nursing, Assiut University. The purpose of the study was explained to the adolescents by the researcher through direct personal communication prior to starting their participation in the study. Also the adolescents were informed that they had the right to agree or disagree to participate in the study. The written consent was taken from all adolescents participating in the study or from their parents and they were informed that data were confidential between them and the researcher and would be used for the purpose of the research only.

\section{Study Intervention}

The researcher first introduced herself to the adolescents and gave them a complete background about the study. Throughout the interview, related information was recorded in the designed sheet depending upon the response of the participant. Every adolescent in the study group received three sessions. The first session included information about thalassemia major as definition, causes, clinical picture, nutrition and exercise. The second session included information about complications and diagnosis about thalassemia major. The third session included discussion and explanation about prevention and treatment of thalassemia major.

Each participant obtained a copy of the brochures that included all the educational material. Finally, after six weeks from the beginning of the educational program, all adolescents in the study and the control groups completed the post-test questionnaire. Self-efficacy of adolescents in the study group was compared to selfefficacy scores of adolescents in the control group.

\section{Field of the work}

Data were collected during the period from the beginning of November 2018 to the end of October 2019. Data were collected during the morning shift for two days each week in the Thalassemia Center. The researcher interviewed each participated adolescent individually to collect data through using the previously mentioned two tools of the study. The time used for filling each sheet ranged between 25: $35 \mathrm{~min}$. 


\section{Statistical Analysis}

The content of each tool was analyzed, categorized and then coded. Data entry and statistical analysis were done using the compatible personal computer through the SPSS statistical Package. Data were presented using descriptive statistics in the form of frequencies and percentages for qualitative variables and mean and standard deviations for quantitative variables. Qualitative variables were compared using Chi-square. A p-value $\leq 0.05$ was considered significant and $\mathrm{p} \leq 0.01$ was considered highly significant.

\section{Results}

Table (1): Demographic characteristics of the studied adolescents with thalassemia major.

\begin{tabular}{|c|c|c|c|c|}
\hline \multirow{2}{*}{ Items } & \multicolumn{2}{|c|}{ Study group $n=(40)$} & \multicolumn{2}{|c|}{ Control group $n=(40)$} \\
\hline & No & $\%$ & No & $\%$ \\
\hline$-12<15$ years & 32 & 80.0 & 28 & 70.0 \\
\hline$-15 \leq 18$ years & 8 & 20.0 & 12 & 30.0 \\
\hline Mean \pm SD & \multicolumn{2}{|c|}{$13.65 \pm 1.58$} & \multicolumn{2}{|c|}{$14.40 \pm 1.93$} \\
\hline Gender & 16 & 40.0 & 22 & 55.0 \\
\hline - Female & 24 & 60.0 & 18 & 45.0 \\
\hline $\begin{array}{ll} & \text { Birth order } \\
& 1^{\text {st }} \text { child }\end{array}$ & 6 & 15.0 & 6 & 15.0 \\
\hline$-2^{\text {nd }}$ child & 24 & 60.0 & 20 & 50.0 \\
\hline$-3^{\text {rd }}$ child & 10 & 25.0 & 12 & 30.0 \\
\hline - $4^{\text {th }}$ or more & 0 & 0.0 & 2 & 5.0 \\
\hline $\begin{array}{ll} & \text { Number of siblings } \\
\text { - One }\end{array}$ & 4 & 10.0 & 4 & 10.0 \\
\hline - Two & 18 & 45.0 & 12 & 30.0 \\
\hline - Three or more & 18 & 45.0 & 24 & 60.0 \\
\hline \multicolumn{5}{|l|}{ Residence } \\
\hline - Rural & 20 & 50.0 & 20 & 50.0 \\
\hline - Urban & 20 & 50.0 & 20 & 50.0 \\
\hline $\begin{array}{l}\text { Educational level } \\
\text { - Basic education }\end{array}$ & 32 & 80.0 & 20 & 50.0 \\
\hline - Secondary & 6 & 15.0 & 18 & 45.0 \\
\hline - University & 2 & 5.0 & 2 & 5.0 \\
\hline
\end{tabular}

Table (2): Percentage distribution of the studied adolescents according to their clinical data $(n=80)$.

\begin{tabular}{|c|c|c|c|c|c|}
\hline \multirow{2}{*}{ Items } & \multicolumn{2}{|c|}{ Study group $n=(40)$} & \multicolumn{2}{|c|}{ Control group $n=(40)$} & \multirow{2}{*}{ P. value } \\
\hline & No & $\%$ & No & $\%$ & \\
\hline \multicolumn{5}{|c|}{ Age at onset of the disease } & \multirow{5}{*}{0.39} \\
\hline$-<6$ months & 2 & 5.0 & 4 & 10.0 & \\
\hline$-6<12$ months & 30 & 75.0 & 28 & 70.0 & \\
\hline$-12<18$ months & 6 & 15.0 & 8 & 20.0 & \\
\hline - $\geq 18$ months & 2 & 5.0 & 0 & 0.0 & \\
\hline \multicolumn{5}{|c|}{ Duration of the disease since first symptoms } & \multirow{4}{*}{$0.00 * *$} \\
\hline$-\quad 5<10$ years & 2 & 5.0 & 0 & 0.0 & \\
\hline - $\quad 10<15$ years & 36 & 90.0 & 28 & 70.0 & \\
\hline$-\quad \geq 15$ years & 2 & 5.0 & 12 & 30.0 & \\
\hline \multicolumn{5}{|c|}{ Onset of chelation therapy } & \multirow{4}{*}{0.90} \\
\hline$-1<3$ years & 2 & 5.0 & 2 & 5.0 & \\
\hline$-3<5$ years & 22 & 55.0 & 20 & 50.0 & \\
\hline$-\quad \geq 5$ years & 16 & 40.0 & 18 & 45.0 & \\
\hline
\end{tabular}




\begin{tabular}{|l|c|c|c|c|c|}
\hline \multirow{2}{*}{ Items } & \multicolumn{2}{|c|}{ Study group n= (40) } & \multicolumn{2}{c|}{ Control group n= (40) } & \multirow{2}{*}{ P. value } \\
\cline { 2 - 5 } & No & $\mathbf{\%}$ & No & \% & \\
\hline Frequency of blood transfusion & & & & & \multirow{2}{*}{$0.02 *$} \\
\hline$-<3$ weeks & 2 & 5.0 & 10 & 25.0 & \\
\hline-3 weeks & 24 & 60.0 & 14 & 35.0 & \\
\hline$->3$ weeks & 14 & 35.0 & 16 & 40.0 & \\
\cline { 1 - 5 } Splenectomy & & & & & \multirow{2}{*}{1.00} \\
\hline$-\quad$ Yes & 26 & 65.0 & 26 & 65.0 & \\
\hline$-\quad$ No & 14 & 35.0 & 14 & 35.0 & \\
\hline
\end{tabular}

*Statistically significant difference ( $p \leq 0.05) * *$ Highly Statistically significant difference $(p \leq 0.01)$

Table (3): Percentage distribution of studied adolescents' family history.

\begin{tabular}{|c|c|c|c|c|c|}
\hline \multirow[t]{2}{*}{ Item } & \multicolumn{2}{|c|}{$\begin{array}{c}\text { Study group } \\
\mathbf{n}=\mathbf{4 0}\end{array}$} & \multicolumn{2}{|c|}{$\begin{array}{c}\text { Control group } n= \\
40\end{array}$} & \multirow[t]{2}{*}{ P.value } \\
\hline & No & $\%$ & No & $\%$ & \\
\hline \multicolumn{5}{|c|}{ Parents' degree of consanguinity } & \multirow{4}{*}{0.37} \\
\hline - No relation & 6 & 15.0 & 4 & 10.0 & \\
\hline - Remote consanguinity & 12 & 30.0 & 18 & 45.0 & \\
\hline - Close consanguinity & 22 & 55.0 & 18 & 45.0 & \\
\hline \multicolumn{5}{|l|}{ Family history } & \multirow{3}{*}{0.052} \\
\hline - Yes & 24 & 60.0 & 32 & 80.0 & \\
\hline$-\mathrm{No}$ & 16 & 40.0 & 8 & 20.0 & \\
\hline \multicolumn{5}{|l|}{ Affected family member } & \multirow{5}{*}{0.32} \\
\hline - Parents & 0 & 0.0 & 0 & 0.0 & \\
\hline - $\quad$ Siblings & 18 & 45.0 & 20 & 50.0 & \\
\hline - Cousins & 6 & 15.0 & 12 & 30.0 & \\
\hline - $\quad$ Other family members & 0 & 0.0 & 0 & 0.0 & \\
\hline
\end{tabular}

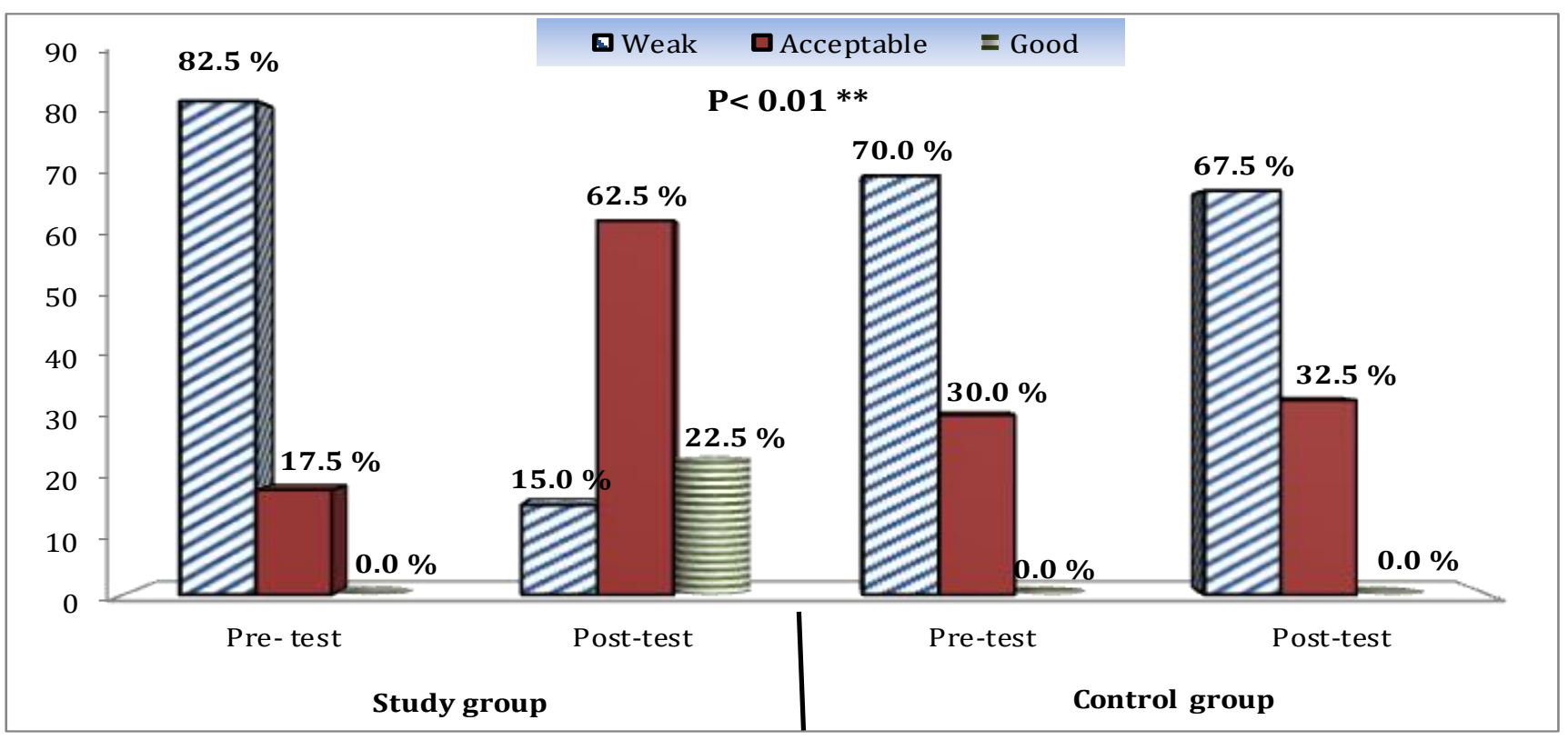

Figure (1): Percentage distribution of knowledge levels among the studied adolescents pre $\&$ post program application. 
Table (4): Percentage Distribution of the studied adolescents' self-efficacy.

\begin{tabular}{|c|c|c|c|c|c|c|c|c|c|}
\hline \multirow{3}{*}{ Items } & \multicolumn{4}{|c|}{ Study group $(n=40)$} & \multicolumn{4}{|c|}{ Control group $(n=40)$} & \multirow{3}{*}{ P. value } \\
\hline & \multicolumn{2}{|c|}{ Pre-test } & \multicolumn{2}{|c|}{ Post-test } & \multicolumn{2}{|c|}{ Pre-test } & \multicolumn{2}{|c|}{ Post-test } & \\
\hline & No & $\%$ & No & $\%$ & No & $\%$ & No & $\%$ & \\
\hline $\begin{array}{l}\text { Daily activities } \\
-\quad \text { Very sure }\end{array}$ & 0 & 0.0 & 0 & 0.0 & 4 & 10.0 & 2 & 5.0 & \multirow{5}{*}{$0.02 *$} \\
\hline - Sure & 0 & 0.0 & 4 & 10.0 & 5 & 12.5 & 3 & 7.5 & \\
\hline - $\quad$ Neither & 11 & 27.5 & 22 & 55.0 & 10 & 25.0 & 13 & 32.5 & \\
\hline - $\quad$ Not sure & 23 & 57.5 & 12 & 30.0 & 19 & 47.5 & 18 & 45.0 & \\
\hline - $\quad$ Not at all sure & 6 & 15.0 & 2 & 5.0 & 2 & 5.0 & 4 & 10.0 & \\
\hline $\begin{array}{l}\text { Overcoming feeling of } \\
\text { sadness } \\
\text { - Very sure }\end{array}$ & 0 & 0.0 & 0 & 0.0 & 0 & 0.0 & 0 & 0.0 & \multirow{5}{*}{0.47} \\
\hline - Sure & 4 & 10.0 & 3 & 7.5 & 10 & 25.0 & 0 & 0.0 & \\
\hline - Neither & 5 & 12.5 & 33 & 82.5 & 15 & 37.5 & 22 & 55.0 & \\
\hline - Not sure & 17 & 42.5 & 4 & 10.0 & 13 & 32.5 & 12 & 30.0 & \\
\hline - Not at all sure & 14 & 35.0 & 0 & 0.0 & 2 & 5.0 & 6 & 15.0 & \\
\hline $\begin{array}{l}\text { Managing the daily life } \\
-\quad \text { Very sure }\end{array}$ & 0 & 0.0 & 0 & 0.0 & 0 & 0.0 & 0 & 0.0 & \multirow{5}{*}{$0.00 * *$} \\
\hline - $\quad$ Sure & 2 & 5.0 & 6 & 15.0 & 1 & 2.5 & 0 & 0.0 & \\
\hline - $\quad$ Neither & 7 & 17.5 & 23 & 57.5 & 12 & 30.0 & 16 & 40.0 & \\
\hline - Not sure & 27 & 67.5 & 11 & 27.5 & 21 & 52.5 & 18 & 45.0 & \\
\hline - $\quad$ Not at all sure & 4 & 10.0 & 0 & 0.0 & 6 & 15.0 & 6 & 15.0 & \\
\hline $\begin{array}{l}\text { Managing symptoms } \\
\text { - Very sure }\end{array}$ & 0 & 0.0 & 0 & 0.0 & 0 & 0.0 & 0 & 0.0 & \multirow{5}{*}{0.25} \\
\hline - Sure & 1 & 2.5 & 9 & 22.5 & 5 & 12.5 & 3 & 7.5 & \\
\hline - Neither & 7 & 17.5 & 21 & 52.5 & 6 & 15.0 & 12 & 30.0 & \\
\hline - Not sure & 24 & 60.0 & 8 & 20.0 & 22 & 55.0 & 17 & 42.5 & \\
\hline - Not at all sure & 8 & 20.0 & 2 & 5.0 & 7 & 17.5 & 8 & 20.0 & \\
\hline $\begin{array}{l}\text { Making friendship } \\
-\quad \text { Very sure } \\
\end{array}$ & 0 & 0.0 & 0 & 0.0 & 0 & 0.0 & 0 & 0.0 & \multirow{5}{*}{$0.00 * *$} \\
\hline - Sure & 3 & 7.5 & 5 & 12.5 & 2 & 5.0 & 7 & 17.5 & \\
\hline - $\quad$ Neither & 6 & 15.0 & 21 & 52.5 & 8 & 20.0 & 6 & 15.0 & \\
\hline - $\quad$ Not sure & 25 & 62.5 & 14 & 35.0 & 18 & 45.0 & 14 & 35.0 & \\
\hline - $\quad$ Not at all sure & 6 & 15.0 & 0 & 0.0 & 12 & 30.0 & 13 & 32.5 & \\
\hline $\begin{array}{l}\text { Dealing with frustration } \\
\text { - Very sure }\end{array}$ & 0 & 0.0 & 0 & 0.0 & 0 & 0.0 & 0 & 0.0 & \multirow{5}{*}{$0.00 * *$} \\
\hline - Sure & 0 & 0.0 & 10 & 25.0 & 0 & 0.0 & 0 & 0.0 & \\
\hline - Neither & 3 & 7.5 & 18 & 45.0 & 11 & 27.5 & 11 & 27.5 & \\
\hline - Not sure & 31 & 77.5 & 12 & 30.0 & 20 & 50.0 & 19 & 47.5 & \\
\hline - Not at all sure & 6 & 15.0 & 0 & 0.0 & 9 & 22.5 & 10 & 25.0 & \\
\hline $\begin{array}{l}\text { School attendance } \\
\text { - Very sure }\end{array}$ & 0 & 0.0 & 0 & 0.0 & 0 & 0.0 & 0 & 0.0 & \multirow{5}{*}{0.19} \\
\hline - Sure & 0 & 0.0 & 3 & 7.5 & 0 & 0.0 & 0 & 0.0 & \\
\hline - Neither & 0 & 0.0 & 15 & 37.5 & 8 & 20.0 & 5 & 12.5 & \\
\hline - Not sure & 8 & 20.0 & 8 & 20.0 & 10 & 25.0 & 11 & 27.5 & \\
\hline - Not at all sure & 32 & 80.0 & 14 & 35.0 & 22 & 55.0 & 24 & 60.0 & \\
\hline $\begin{array}{l}\text { Paying attention } \\
\text { - Very sure }\end{array}$ & 0 & 0.0 & 0 & 0.0 & 0 & 0.0 & 0 & 0.0 & \multirow{4}{*}{$0.00 * *$} \\
\hline - Sure & 4 & 10.0 & 8 & 20.0 & 1 & 2.5 & 0 & 0.0 & \\
\hline - Neither & 0 & 0.0 & 16 & 40.0 & 7 & 17.5 & 8 & 20.0 & \\
\hline - Not sure & 14 & 35.0 & 14 & 35.0 & 12 & 30.0 & 11 & 27.5 & \\
\hline
\end{tabular}


Sadek et al.,

\begin{tabular}{|c|c|c|c|c|c|c|c|c|c|}
\hline \multirow{3}{*}{ Items } & \multicolumn{4}{|c|}{ Study group $(n=40)$} & \multicolumn{4}{|c|}{ Control group $(n=40)$} & \multirow{4}{*}{ P. value } \\
\hline & \multicolumn{2}{|c|}{ Pre-test } & \multicolumn{2}{|c|}{ Post-test } & \multicolumn{2}{|c|}{ Pre-test } & \multicolumn{2}{|c|}{ Post-test } & \\
\hline & No & $\%$ & No & $\%$ & No & $\%$ & No & $\%$ & \\
\hline - Not at all sure & 22 & 55.0 & 2 & 5.0 & 20 & 50.0 & 21 & 52.5 & \\
\hline $\begin{array}{l}\text { Accomplishing goals } \\
\text { - Very sure }\end{array}$ & 0 & 0.0 & 0 & 0.0 & 0 & 0.0 & 1 & 2.5 & \multirow{5}{*}{$0.00 * *$} \\
\hline - Sure & 4 & 10.0 & 6 & 15.0 & 3 & 7.5 & 0 & 0.0 & \\
\hline - Neither & 4 & 10.0 & 14 & 35.0 & 2 & 5.0 & 5 & 12.5 & \\
\hline - Not sure & 4 & 10.0 & 10 & 25.0 & 9 & 22.5 & 10 & 25.0 & \\
\hline - Not at all sure & 28 & 70.0 & 10 & 25.0 & 26 & 65.0 & 24 & 60.0 & \\
\hline
\end{tabular}

*Statistically significant difference $(\mathrm{p} \leq 0.05) * *$ Highly Statistically significant difference $(\mathrm{p} \leq 0.01)$

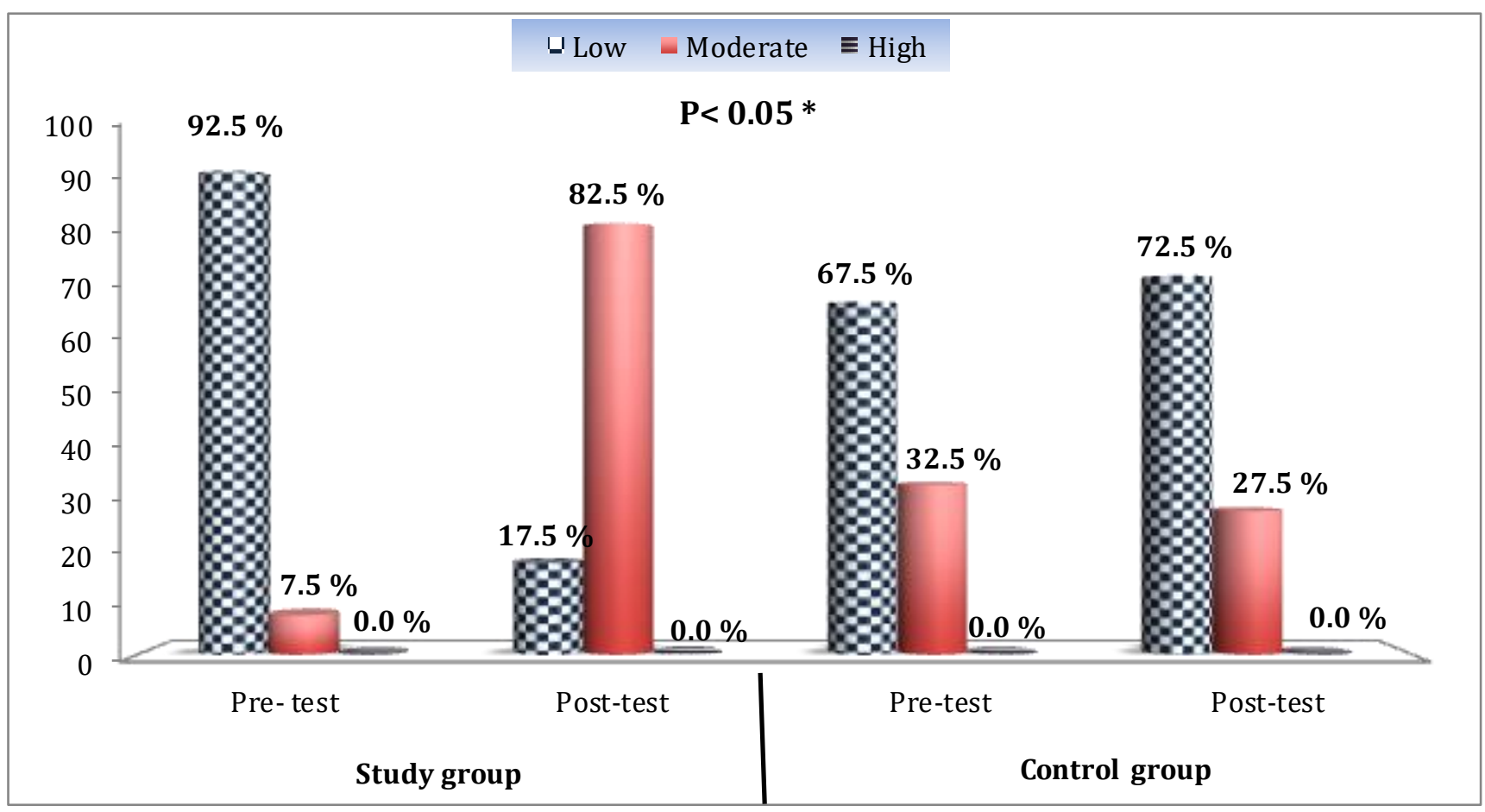

Figure (2): Comparison of the studied adolescents' self-efficacy levels pre and post program application. 
Table (5): Relation between the studied adolescents' knowledge level and demograpic characteristics.

\begin{tabular}{|c|c|c|c|c|c|c|c|c|c|c|c|c|c|c|c|c|c|c|c|c|c|c|c|c|c|}
\hline \multirow{5}{*}{ Item } & \multicolumn{24}{|c|}{ Knowledge level } & \multirow{5}{*}{ P. value } \\
\hline & \multicolumn{12}{|c|}{ Study group $n=40$} & \multicolumn{12}{|c|}{ Control group $n=40$} & \\
\hline & \multicolumn{6}{|c|}{ Pre-test } & \multicolumn{6}{|c|}{ Post-test } & \multicolumn{6}{|c|}{ Pre-test } & \multicolumn{6}{|c|}{ Post-test } & \\
\hline & \multicolumn{2}{|c|}{ Weak } & \multicolumn{2}{|c|}{ Acceptable } & \multicolumn{2}{|c|}{ Good } & \multicolumn{2}{|c|}{ Weak } & \multicolumn{2}{|c|}{ Acceptable } & \multicolumn{2}{|c|}{ Good } & \multicolumn{2}{|c|}{ Weak } & \multicolumn{2}{|c|}{ Acceptable } & \multicolumn{2}{|c|}{ Good } & \multicolumn{2}{|c|}{ Weak } & \multicolumn{2}{|c|}{ Acceptable } & \multicolumn{2}{|c|}{ Good } & \\
\hline & No & $\%$ & No & $\%$ & No & $\%$ & No & $\%$ & No & $\%$ & No & $\%$ & No & $\%$ & No & $\%$ & No & $\%$ & No & $\%$ & No & $\%$ & No & $\%$ & \\
\hline $\begin{array}{l}\text { Age } \\
-\quad 12<15 \\
\end{array}$ & 28 & 87.5 & 4 & 12.5 & 0 & 0.0 & 6 & 18.8 & 20 & 62.4 & 6 & 18.8 & 22 & 78.6 & 6 & 21.4 & 0 & 0.0 & 21 & 75.0 & 7 & 25.0 & 0 & 0.0 & \multirow[t]{2}{*}{0.06} \\
\hline - $16 \leq 18$ & 5 & 62.5 & 3 & 37.5 & 0 & 0.0 & 0 & 0.0 & 5 & 62.5 & 3 & 37.5 & 6 & 50.0 & 6 & 50.0 & 0 & 0.0 & 6 & 50.0 & 6 & 50.0 & 0 & 0.0 & \\
\hline $\begin{array}{r}\text { Gender } \\
-\quad \text { Male } \\
\end{array}$ & 12 & 75.0 & 4 & 25.0 & 0 & 0.0 & 2 & 12.5 & 9 & 56.2 & 5 & 31.2 & 16 & 72.7 & 6 & 27.3 & 0 & 0.0 & 17 & 77.3 & 5 & 22.7 & 0 & 0.0 & \multirow[t]{2}{*}{0.57} \\
\hline - Female & 21 & 87.5 & 3 & 12.5 & 0 & 0.0 & 4 & 16.7 & 16 & 66.7 & 4 & 16.7 & 12 & 66.7 & 6 & 33.3 & 0 & 0.0 & 10 & 55.6 & 8 & 44.4 & 0 & 0.0 & \\
\hline \begin{tabular}{l}
\multicolumn{1}{c}{ Birth order } \\
$-\quad 1^{\text {st }}$ child \\
\end{tabular} & 5 & 83.3 & 1 & 16.7 & 0 & 0.0 & 0 & 0.0 & 4 & 66.7 & 2 & 33.3 & 6 & 100.0 & 0 & 0.0 & 0 & 0.0 & 5 & 83.3 & 1 & 16.7 & 0 & 0.0 & \multirow{4}{*}{0.14} \\
\hline$-2^{\text {nd }}$ child & 20 & 83.3 & 4 & 16.7 & 0 & 0.0 & 4 & 16.7 & 13 & 54.2 & 7 & 29.2 & 13 & 65.0 & 7 & 35.0 & 0 & 0.0 & 14 & 70.0 & 6 & 30.0 & 0 & 0.0 & \\
\hline - $3^{\text {rd }}$ child & 8 & 80.0 & 2 & 20.0 & 0 & 0.0 & 2 & 20.0 & 8 & 80.0 & 0 & 0.0 & 7 & 58.3 & 5 & 41.7 & 0 & 0.0 & 6 & 50.0 & 6 & 50.0 & 0 & 0.0 & \\
\hline$-4^{\text {th }}$ or more & 0 & 0.0 & 0 & 0.0 & 0 & 0.0 & 0 & 0.0 & 0 & 0.0 & 0 & 0.0 & 2 & 100.0 & 0 & 0.0 & 0 & 0.0 & 2 & 100.0 & 0 & 0.0 & 0 & 0.0 & \\
\hline $\begin{array}{l}\text { Number of siblings } \\
-\quad \text { One }\end{array}$ & 4 & $100.0 \mid$ & 0 & 0.0 & 0 & 0.0 & 0 & 0.0 & 4 & 100.0 & 0 & 0.0 & 2 & 50.0 & 2 & 50.0 & 0 & 0.0 & 2 & 50.0 & 2 & 50.0 & 0 & 0.0 & \multirow{3}{*}{0.28} \\
\hline $\begin{array}{ll}- & \text { Two } \\
\end{array}$ & 15 & 83.3 & 3 & 16.7 & 0 & 0.0 & 2 & 11.1 & 10 & 55.6 & 6 & 33.3 & 9 & 75.0 & 3 & 25.0 & 0 & 0.0 & 9 & 75.0 & 3 & 25.0 & 0 & 0.0 & \\
\hline - $\quad$-Three or more & 14 & 77.8 & 4 & 22.2 & 0 & 0.0 & 4 & 22.2 & 11 & 61.1 & 3 & 16.7 & 17 & 70.8 & 7 & 29.2 & 0 & 0.0 & 16 & 66.7 & 8 & 33.3 & 0 & 0.0 & \\
\hline $\begin{array}{l}\text { Residence } \\
-\quad \text { Rural } \\
\end{array}$ & 18 & 90.0 & 2 & 10.0 & 0 & 0.0 & 4 & 20.0 & 13 & 65.0 & 3 & 15.0 & 18 & 90.0 & 2 & 10.0 & 0 & 0.0 & 19 & 95.0 & 1 & 5.0 & 0 & 0.0 & \multirow[t]{2}{*}{$0.01 * *$} \\
\hline - $\quad$ Urban & 15 & 75.0 & J & 25.0 & 0 & 0.0 & 2 & 10.0 & 12 & 60.0 & 0 & 30.0 & 10 & 50.0 & 10 & 50.0 & 0 & 0.0 & 8 & 40.0 & 12 & 60.0 & 0 & 0.0 & \\
\hline $\begin{array}{l}\text { Educational level } \\
-\quad \text { Basic education } \\
\end{array}$ & 28 & 87.5 & 4 & 12.5 & 0 & 0.0 & 6 & 18.8 & 20 & 62.5 & 6 & 18.8 & 14 & 70.0 & 6 & 30.0 & 0 & 0.0 & 16 & 80.0 & 4 & 20.0 & 0 & 0.0 & \\
\hline - Secondary & 3 & 50.0 & 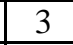 & 50.0 & 0 & 0.0 & 0 & 0.0 & 3 & 50.0 & $J$ & 50.0 & 14 & 77.8 & 4 & 22.2 & 0 & 0.0 & 11 & 61.1 & 7 & 38.9 & 0 & 0.0 & 21 \\
\hline - University & 2 & 100.0 & 0 & 0.0 & 0 & 0.0 & 0 & 0.0 & 2 & 100.0 & 0 & 0.0 & 0 & 0.0 & 2 & 100.0 & 0 & 0.0 & 0 & 0.0 & 2 & 100.0 & 0 & 0.0 & \\
\hline
\end{tabular}


Table (6): Relation between the studied adolescents' self-efficacy level and their demographic characteristics.

\begin{tabular}{|c|c|c|c|c|c|c|c|c|c|c|c|c|c|c|c|c|c|c|c|c|c|c|c|c|c|}
\hline \multirow{5}{*}{ Item } & \multicolumn{24}{|c|}{ Self-efficacy level } & \multirow{5}{*}{ P.value } \\
\hline & \multicolumn{12}{|c|}{ Study group $n=40$} & \multicolumn{12}{|c|}{ Control group $n=40$} & \\
\hline & \multicolumn{6}{|c|}{ Pre-test } & \multicolumn{6}{|c|}{ Post-test } & \multicolumn{6}{|c|}{ Pre-test } & \multicolumn{6}{|c|}{ Post-test } & \\
\hline & \multicolumn{2}{|c|}{ Low } & \multicolumn{2}{|c|}{ Moderate } & \multicolumn{2}{|c|}{ High } & \multicolumn{2}{|c|}{ Low } & \multicolumn{2}{|c|}{ Moderate } & \multicolumn{2}{|c|}{ High } & \multicolumn{2}{|c|}{ Low } & \multicolumn{2}{|c|}{ Moderate } & \multicolumn{2}{|c|}{ High } & \multicolumn{2}{|c|}{ Low } & \multicolumn{2}{|c|}{ Moderate } & \multicolumn{2}{|c|}{ High } & \\
\hline & No & $\%$ & No & $\%$ & No & $\%$ & No & $\%$ & No & $\%$ & No & $\%$ & No & $\%$ & No & $\%$ & No & $\%$ & No & $\%$ & No & $\%$ & No & $\%$ & \\
\hline $\begin{array}{r}\quad \text { Age } \\
-\quad 12<15 \\
\end{array}$ & 31 & 96.9 & 1 & 3.1 & 0 & 0.0 & 7 & 21.9 & 25 & 78.1 & 0 & 0.0 & 20 & 71.4 & 8 & 28.6 & 0 & 0.0 & 21 & 75.0 & 7 & 25.0 & 0 & 0.0 & \multirow[t]{2}{*}{0.09} \\
\hline$-\quad 16 \leq 18$ & 6 & 75.0 & 2 & 25.0 & 0 & 0.0 & 0 & 0.0 & 8 & 100.0 & 0 & 0.0 & 7 & 58.3 & 5 & 41.7 & 0 & 0.0 & 8 & 66.7 & 4 & \begin{tabular}{|l|}
33.3 \\
\end{tabular} & 0 & 0.0 & \\
\hline $\begin{array}{l}\text { Gender } \\
\text { - Male }\end{array}$ & 13 & 81.2 & 3 & 18.8 & 0 & 0.0 & 2 & 12.5 & 14 & 87.5 & 0 & 0.0 & 15 & 68.2 & 7 & 31.8 & 0 & 0.0 & 14 & 63.6 & 8 & 36.4 & 0 & 0.0 & \multirow{2}{*}{0.16} \\
\hline - $\quad$ Female & 24 & 100.0 & 0 & 0.0 & 0 & 0.0 & 5 & 20.8 & 19 & 79.2 & 0 & 0.0 & 12 & 66.7 & 6 & 33.3 & 0 & 0.0 & 15 & 83.3 & 3 & 16.7 & 0 & 0.0 & \\
\hline $\begin{array}{ll} & \text { Birth order } \\
-\quad & 1^{\text {st }} \text { child }\end{array}$ & 6 & 100.0 & 0 & 0.0 & 0 & 0.0 & 0 & 0.0 & 6 & 100.0 & 0 & 0.0 & 6 & 100.0 & 0 & 0.0 & 0 & 0.0 & 6 & 100.0 & 0 & 0.0 & 0 & 0.0 & \multirow{4}{*}{0.15} \\
\hline - $2^{\text {nd }}$ child & 21 & 87.5 & 3 & 12.5 & 0 & 0.0 & 4 & 16.7 & 20 & 83.3 & 0 & 0.0 & \begin{tabular}{|l|}
12 \\
\end{tabular} & 60.0 & 8 & 40.0 & 0 & 0.0 & 14 & 70.0 & 6 & 30.0 & 0 & 0.0 & \\
\hline$-3^{\text {rd }}$ child & 10 & 100.0 & 0 & 0.0 & 0 & 0.0 & 3 & 30.0 & 7 & 70.0 & 0 & 0.0 & 8 & 66.7 & 4 & 33.3 & 0 & 0.0 & 9 & 75.0 & 3 & 25.0 & 0 & 0.0 & \\
\hline - $4^{\text {th }}$ or more & 0 & 0.0 & 0 & 0.0 & 0 & 0.0 & 0 & 0.0 & 0 & 0.0 & 0 & 0.0 & 1 & 50.0 & 1 & 50.0 & 0 & 0.0 & 0 & 0.0 & 2 & 100.0 & 0 & 0.0 & \\
\hline $\begin{array}{l}\text { Number of siblings } \\
-\quad \text { One }\end{array}$ & 4 & 100.0 & 0 & 0.0 & 0 & 0.0 & 2 & 50.0 & 2 & 50.0 & 0 & 0.0 & 0 & 0.0 & 4 & 100.0 & 0 & 0.0 & 2 & 50.0 & 2 & 50.0 & 0 & 0.0 & \multirow{3}{*}{0.36} \\
\hline - $\quad$ Two & 18 & 100.0 & 0 & 0.0 & 0 & 0.0 & 3 & 16.7 & 15 & 83.3 & 0 & 0.0 & 10 & 83.3 & 2 & 16.7 & 0 & 0.0 & 10 & \begin{tabular}{|l|}
83.3 \\
\end{tabular} & 2 & 16.7 & 0 & 0.0 & \\
\hline - $\quad$ Three or more & 15 & 83.3 & 3 & 16.7 & 0 & 0.0 & 2 & 11.1 & 16 & 88.9 & 0 & 0.0 & 17 & 70.0 & 7 & 29.2 & 0 & 0.0 & 17 & \begin{tabular}{|l|}
70.8 \\
\end{tabular} & 7 & 29.2 & 0 & 0.0 & \\
\hline $\begin{array}{l}\text { Residence } \\
\text { - } \quad \text { Rural } \\
\end{array}$ & 17 & 85.0 & 3 & 15.0 & 0 & 0.0 & 1 & 5.0 & 19 & 95.0 & 0 & 0.0 & 16 & 80.0 & 4 & 20.0 & 0 & 0.0 & 16 & 80.0 & 4 & 20.0 & 0 & 0.0 & \multirow[t]{2}{*}{1.00} \\
\hline - $\quad$ Urban & 20 & 100.0 & 0 & 0.0 & 0 & 0.0 & 6 & 30.0 & 14 & 70.0 & 0 & 0.0 & 11 & 55.0 & 9 & 45.0 & 0 & 0.0 & 13 & \begin{tabular}{|l|}
65.0 \\
\end{tabular} & 7 & 35.0 & 0 & 0.0 & \\
\hline $\begin{array}{c}\text { Educational level } \\
\text {-Basic education }\end{array}$ & 31 & 96.9 & 1 & 3.1 & 0 & 0.0 & 7 & 21.9 & 25 & 78.1 & 0 & 0.0 & 14 & 70.0 & 6 & 30.0 & 0 & 0.0 & 16 & 80.0 & 4 & 20.0 & 0 & 0.0 & \\
\hline - $\quad$ Secondary & 4 & 66.7 & 2 & 33.3 & 0 & 0.0 & 0 & 0.0 & 6 & 100.0 & 0 & 0.0 & 13 & 72.2 & 5 & 27.8 & 0 & 0.0 & 13 & 72.2 & 5 & 27.8 & 0 & 0.0 & 0.08 \\
\hline - University & 2 & 100.0 & 0 & 0.0 & 0 & 0.0 & 0 & 0.0 & 2 & 100.0 & 0 & 0.0 & 0 & 0.0 & 2 & 100.0 & 0 & 0.0 & 0 & 0.0 & 2 & 100.0 & 0 & 0.0 & \\
\hline
\end{tabular}


Table (7): Relation between the studied adolescents' knowledge level and their self-efficacy level.

\begin{tabular}{|c|c|c|c|c|c|c|c|c|c|c|c|c|c|c|c|c|c|c|c|c|c|c|c|c|c|}
\hline \multirow{5}{*}{$\begin{array}{c}\text { Knowledge } \\
\text { Level }\end{array}$} & \multicolumn{24}{|c|}{ Self-efficacy level } & \multirow{5}{*}{ P.value } \\
\hline & \multicolumn{12}{|c|}{ Study group $n=40$} & \multicolumn{12}{|c|}{ Control group $n=40$} & \\
\hline & \multicolumn{6}{|c|}{ Pre-test } & \multicolumn{6}{|c|}{ Post-test } & \multicolumn{6}{|c|}{ Pre-test } & \multicolumn{6}{|c|}{ Post-test } & \\
\hline & \multicolumn{2}{|c|}{ Low } & \multicolumn{2}{|c|}{ Moderate } & \multicolumn{2}{|c|}{ High } & \multicolumn{2}{|c|}{ Low } & \multicolumn{2}{|c|}{ Moderate } & \multicolumn{2}{|c|}{ High } & \multicolumn{2}{|c|}{ Low } & \multicolumn{2}{|c|}{ Moderate } & \multicolumn{2}{|c|}{ High } & \multicolumn{2}{|c|}{ Low } & \multicolumn{2}{|c|}{ Moderate } & \multicolumn{2}{|c|}{ High } & \\
\hline & No & $\%$ & No & $\%$ & No & $\%$ & No & $\%$ & No & $\%$ & No & $\%$ & No & $\%$ & \begin{tabular}{l|l} 
No & \\
\end{tabular} & $\%$ & No| & $\%$ & No & $\%$ & \begin{tabular}{|l|} 
No \\
\end{tabular} & $\%$ & No & $\%$ & \\
\hline Weak & 32 & 97.0 & 1 & 3.0 & 0 & 0.0 & 0 & 0.0 & 6 & 100.0 & 0 & 0.0 & 23 & 82.1 & 5 & 17.9 & 0 & 0.0 & 21 & 77.8 & 6 & 22.2 & 0 & 0.0 & \\
\hline Acceptable & 5 & 71.4 & 2 & 28.6 & 0 & 0.0 & 7 & 28.0 & 18 & 72.0 & 0 & 0.0 & 4 & 33.3 & 8 & 66.7 & 0 & 0.0 & 8 & 61.5 & 5 & 38.5 & 0 & 0.0 & $0.00 * *$ \\
\hline Good & 0 & 0.0 & 0 & 0.0 & 0 & 0.0 & 0 & 0.0 & 9 & 100.0 & 0 & 0.0 & 0 & 0.0 & 0 & 0.0 & 0 & 0.0 & 0 & 0.0 & 0 & 0.0 & 0 & 0.0 & \\
\hline
\end{tabular}

** Highly Statistically significant difference $(p \leq 0.01)$ 
Table (1): Represents demographic characteristics of the studied adolescents with thalassemia major. The results showed that $80.0 \%$ and $70.0 \%$ of the adolescents in the study and the control groups respectively aged between twelve and less than fifteen years old with a mean \pm SD of $13.65 \pm 1.58$ for the study group and $14.40 \pm 1.93$ years for the control group. According to their gender, three-fifths $(60.0 \%)$ of the adolescents of the study group were females while, more than half $(55.0 \%)$ of the adolescents in the control group were males.

Regards the birth order, three-fifths $(60.0 \%)$ of the adolescents in the study group and half $(50.0 \%)$ of the adolescents in the control group ranked second in their families. The results revealed that $45.0 \%$ and $60.0 \%$ of the adolescents in the study and the control groups respectively had three or more siblings. Regarding the educational level, the majority $(80.0 \%)$ of the study group and half (50.0 $\%$ ) of the control group had basic education.

Table (2): Indicates percentage distribution of the studied adolescents according to their clinical data. Regards age of onset of the disease, it was between 6 $<12$ months old in the highest percentages $(75.0 \%$ for the study group and $70.0 \%$ for the control group) of the studied adolescents. According to duration of the disease since first symptoms, it ranged from 10 up to 15 years in the majority of both the study and the control groups $(90.0 \%$ and $70.0 \%$ respectively). More than half $(55.0 \%)$ of the adolescents in the study group and half $(50.0 \%)$ of the adolescents in the control group started the chelation therapy when they were 3 to 5 years old.

Furthermore, the results indicated that $60.0 \%$ of the adolescents in the study group received blood transfusion once every three weeks while $40.0 \%$ of the adolescents in the control group had blood transfusion once every more than three weeks. Also, more than two-thirds $(65.0 \%)$ of the adolescents of both groups had splenectomy. Besides, there was a highly statistically significant difference between adolescents in the study group and the control group as regards duration of the disease since first symptoms $(p=0.00)$. Besides, a statistically significant difference was present between the study and the control groups regarding frequency of blood transfusion $(\mathrm{P}=0.02)$.

Table (3): Demonstrates the percentage distribution of family history of thalassemia major among the studied adolescents. According to parents' degree of consanguinity, more than half $(55.0 \%)$ of the adolescents' parents in the study group had close consanguinity, while more than two-fifths $(45.0 \%)$ in the control group had close consanguinity. The present study noted that less than two-thirds (60.0 $\%$ ) of the adolescents in the study group and the majority $(80.0 \%)$ of the control group had positive family history of thalassemia major. More than twofifths $(45.0 \%)$ of the adolescents in the study group and half $(50.0 \%)$ of the adolescents in the control group had affected siblings with thalassemia major. No statistically significant differences were found between the study and the control groups regarding all family history items.

Figure (1): Shows percentage distribution of knowledge levels among the studied adolescents pre \& post program application. Regarding pre-test knowledge levels, the results illustrated that the majority of the adolescents in the study group (82.5 $\%)$ vs. $70.0 \%$ for the control group had weak knowledge levels. Regarding post-test knowledge levels, the results found that less than two-thirds $(62.5 \%)$ of the adolescents in the study group had acceptable knowledge levels while more than twothirds $(67.5 \%)$ of the adolescents in the control group had weak knowledge levels. There was a highly statistically significant difference between the study and the control groups regarding their knowledge scores $(\mathrm{p}=0.01)$.

Table (4): Represents percentage distribution of the studied adolescents' self-efficacy. The results revealed a statistically significant difference between the study and the control group regarding daily activities $(\mathrm{P}=0.02)$. Besides, there were highly statistically significant differences between both groups regarding managing the daily life, making friendship, dealing with frustration, paying attention and accomplishing goals $(\mathrm{P}=0.00)$.

Figure (2): Shows comparison of the studied adolescents' self-efficacy levels pre and post program application. Regarding pre-test self-efficacy levels, the results indicated that $(92.5 \%$ vs. $67.5 \%)$ of the adolescents in the study and the control groups had low self-efficacy levels. In post-test, $(82.5 \%)$ of the adolescents in the study group had moderate self-efficacy levels while less than threequarters $(72.5 \%)$ of the adolescents in the control group had low self-efficacy levels. The results showed that there was a statistically significant difference between the two groups regarding selfefficacy scores $(\mathrm{p}=0.05)$.

Table (5): Illustrates the relation between the studied adolescents' knowledge level and their demographic characteristics. The present study revealed a highly statistically significant difference between the study and the control group regards their residence $(\mathrm{P}=0.01)$. As revealed from the table, urban residents had higher knowledge levels compared to rural residents (in study group; $75.0 \%$, $25.0 \%, 0.0 \%$ vs. $90.0 \%, 10.0 \%, 0.0 \%$ for the pretest while in the post-test $10.0 \%, 60.0 \%, 30.0 \%$ vs. 
$20.0 \%, 65.0 \%, 15.0 \%$ for weak, acceptable and good levels respectively).

Table (6): Represents relation between the studied adolescents' self-efficacy level and their demographic characteristics. The present study demonstrated that no statistically significant differences were found between the studied adolescents' self-efficacy as regards all demographic items.

Table (7): Illustrates the relation between the studied adolescents' knowledge level and their selfefficacy level. The results showed that there was a highly statistically significant difference between the studied adolescents' knowledge level and their selfefficacy level $(p=0.00)$. As revealed from the table, higher self-efficacy levels were associated with good knowledge level.

\section{Discussion}

Adolescents with thalassemia major face many challenges in adjusting to their illness which predispose them for poor longitudinal outcome. One of the most important modifiable constructs for adolescent adjustment is self-efficacy. Coping with thalassemia major is best when the adolescent demonstrates high self-efficacy, takes charge of life situations, has a strong belief in his ability to achieve goals and has a strong expectation of being successful with the outcome (Parhiz et al., 2016).

The present study results revealed the highest percentage of the adolescents with thalassemia major in both the study and the control groups was aged between twelve and less than fifteen years old with a mean age of $13.65 \pm 1.58$ and $14.40 \pm 1.93$ years for the study and the control groups respectively. This is quite different from results of a study by Borimnejad et al., (2017), who presented the mean and standard deviation of the adolescents' age were $16 \pm 1.9$ in the study group and $15.2 \pm 2$ in the control group.

Regarding birth order, the current study results indicated that three-fifths of the adolescents of the study group were the second child, while half the adolescents of the control group were the second child. This result disagrees with results of Shareef \& Obaid (2015), which suggested that more than two-thirds of the studied adolescents were the first child in their families.

The current study revealed that disease onset in the highest percentage of the studied adolescents was between six up to twelve months. This goes in the same line with results of Hussein \& Mansour (2015), who indicated that more than two-thirds of the studied adolescents were infants at the age of diagnosis of thalassemia. This can be attributed to the highly probability for thalassemia major to be recognized during the latter half of the first year due to the high tendency of the disease to be more symptomatic.

More than half of the adolescents in the study group and an exact half of the adolescents in the control group started their chelation therapy when they were $3-<5$ years old. This is different from results of Shareef \& Obaid (2015), who indicated that the majority of the studied adolescents started the iron chelating therapy after five years old. This difference between results of the two studies may be attributed, from the researcher's point of view, to difference between the study subjects regarding their frequency of blood transfusion and their annual amount of blood administered which in turn affects the age of starting chelation therapy.

Moreover, the study results reported that three-fifths of adolescents in the study group had blood transfusion once every 3 weeks, while two-fifths of adolescents in the control group had blood transfusion once every more than 3 weeks. This is quite similar to results of a study conducted by Hussein \& Mansour (2015), which revealed that more than three-quarters of the studied adolescents were taking blood transfusion 1-2 times monthly. This indicates the essentiality of regular blood transfusion for the survival of affected children with thalassemia major.

Furthermore, the current study showed that more than two-thirds of the studied adolescents in both the study and the control groups had splenectomy. Results of the current study support the evidence that patients with thalassemia major often require splenectomy to limit the number of required transfusions especially if the annual transfusion requirement increases to or more than 200 to 220 $\mathrm{mL} \mathrm{RBCs} / \mathrm{kg} /$ year with a hematocrit value of $70 \%$.

Comparing findings of this study with other studies, different results was reported by results of Hussein \& Mansour (2015), which revealed that nearly onethird of the studied patients had splenectomy. This contradiction between results of the study and results of the current study may be due to difference between the study subjects' amount of annual blood transfusion.

As regards consanguinity degree, more than half and more than two-fifths of parents of the adolescents in the study and the control groups were closely related as noted from the results of the current study. Consanguineous marriages are common in Egypt due to cultural and economic factors which support relative marriages.

In addition, a highly statistically significant difference was found between the study and the control groups regarding self-efficacy scores. This result goes along with the study done by 
Moghadam et al., (2016). Similarly, a study conducted by Masinaeinezhad et al., (2018) showed a statistically significant difference of selfefficacy score between the two groups before and after intervention. This, from the researcher's opinion, signifies the effect of the educational program on improving the self-efficacy of the affected adolescents with thalassemia major.

The current study revealed a highly statistically significant relation between the studied adolescents' knowledge level regarding their residence. Higher knowledge levels were noted in the urban residents. Difference between the knowledge level of rural and urban adolescents may be attributed to difference of availability and distribution of health institutes and centers responsible for providing health education between rural and urban areas. This contradicts results of Kourorian et al., (2014), who showed a statistically insignificant relation between the knowledge and gender or any other demographic parameters.

As revealed from the current study results, no statistically significant difference was found between the studied adolescents' self-efficacy level and their demographic data. This agrees with findings of Hussein \& Mansour (2015), who indicated a non-significant difference between selfefficacy and items of demographic aspects as age and gender.

The present study revealed that higher self-efficacy levels were noted in the studied adolescents with good knowledge levels. This ensures effectiveness of the educational program in increasing the studied adolescents' knowledge regarding thalassemia major. This is consistent with a study done by Abu Samra et al., (2015), who concluded that there was a positive effect of the educational program in improving Beta-thalassemia adolescents' knowledge regarding thalassemia.

\section{Conclusion}

The present study highlighted the effectiveness of the educational program on self-efficacy of adolescents with thalassemia major.

\section{Recommendations}

Based upon findings of the current study, the following recommendations are suggested;

1- Health education programs are recommended on a regular basis for adolescents with thalassemia major to increase their knowledge about the disease and how to cope with it.

2- Raising the public awareness of medical hazards of consanguineous marriage and benefits of pre-marriage tests are highly recommended through various mass-media.

3- Adolescents with thalassemia major need to participate in common social group activities and empowerment programs to enhance their self-efficacy.

\section{References}

1. Abu Samra, O., Auda, W., Kamhawy, H., \& Al-Tonbary, Y., (2015): Impact of Educational Programme regarding Chelation Therapy on the Quality of Life for BThalassemia Major Children. Journal of Hematology, 20 (5): 297- 303.

2. Al-Kherbash, H., Al-AwdI, A., \& Hassan, N., (2018): Pattern and Clinical Profile of Thalassemia among Pediatric Patients attending the Yemeni Society Centers for Thalassemia and Genetic Blood Disorders in Yemen. The Scientific Journal of Al-Azhar Medical Faculty of Girls, 1 (2): 43-56.

3. Ankush, A., Silveira, M., Talwadker, Y., and Souza, J., (2019): Quality of Life in Children with Thalassemia Major Following up at A Tertiary Care Center in India (GOTQoL). International Journal of Contemporary Pediatrics, 6 (1): 168-75.

4. Basu, M., (2015): A Study on Knowledge, Attitude and Practice about Thalassemia among General Population in Outpatient Department at A Tertiary Care Hospital Of Kolkata. Journal of Preventive Medicine and Holistic Health, 1 (1): 5- 12.

5. Biswas, B., Naskar, N., Basu, R., Dasgupta, A., Paul, B., \& Basu, K., (2019): Knowledge of the Caregivers of Thalassemic Children Regarding Thalassemia: A Cross-sectional Study In A Tertiary Care Health Facility of Eastern India. Iraqi Journal of Hematology, 7 (2): 49- 54 .

6. Borimnejad, L., Parvizy, S., Haghaani, H., \& Sheibani, B., (2017): The Effect of Family-Centered Empowerment Program on Self-Efficacy of Adolescents with Thalassemia Major: A Randomized Controlled Clinical Trial. 6 (1): 29-38.

7. Edwards, R., Telfair, J., Cecil, H., \& Lenoci, J., (2001): Reliability and Validity of A Self-Efficacy Instrument Specific to Sickle Cell Disease, Behaiour Research and Therapy, 38 (9): 951- 63.

8. Elsayed, L., \& Abd El-Gawad, S., (2015): Nursing Guidelines for Children Suffering from Beta Thalassemia. Journal of Education and Health Promotion, 5 (4): 131- 35. 
9. Gupta, P., (2017): Essential Pediatric Nursing. 4th ed., Ch. 16, CBS Publishing \& Distribution Pvt Ltd, New Delhi, India. Pp. 287- 89.

10. Hussein, M., \& Mansour K., (2015): SelfEfficacy among Thalassemic Adults Patients at Hereditary Anemia's Centers in Baghdad. IOSR Journal of Nursing and Health Science (IOSR-JNHS), 4 (5): 75-82.

11. Kourorian, Z., Azarkeivan, A., Hajibeigi, B., Oshidari, A., \& Shirkavnd, A., (2014): The Effect of Knowledge, Attitude and Practice on the Function of Thalassemic Patients. Iranian Journal of Blood and Cancer, 6 (4): 177- 81.

12. Lai, J., Luo, J., Luo, L., Liu, R., Liu, Z., \& Zhao. Z., (2017): SOX6 Downregulation Induces $\gamma$-Globin in Human $\beta$-Thalassemia Major Erythroid Cells. BioMed Research International, 1 (2): 1 - 6.

13. Madmoli, Y., Salimi, M., Madmoli, M., Davoodi, N., Rostami, F., HeidariSoureshjani, R., \& Yousefi, N., (2019): The Relationship between Spiritual Well-being and Family Cohesion in Patients with Thalassemia Major. Journal of Research in Medical and Dental Science, 7 (2): 203- 9.

14. Masinaeinezhad,

N., Abdollahimohammad, A., Bonjar, A., \& Allahyari, J., (2018): The Effect of SelfCare Education Based on Orem's Model on Self-Efficacy of patients with Beta Thalassemia Major. La Prensa Medica Argentina, 104 (4): 1-4.

15. Moghadam, M., Nourisancho, H., Shahdadi, H., Shahraki, S., Azarkish, B., and Balouchi, A., (2016): Effects of HomeCare Training on the Self-Efficacy of Patients with Beta Thalassemia Major. Mater Sociomedical Journal, 28 (5): 357-60.

16. Parhiz, Z., Birjandi, M., Khazaie, T., \& Sharifzadeh, G., (2016): The Effects of an Empowerment Program on the Knowledge, Self-Efficacy, Self-Esteem, and Attitudes of Mothers of Preterm Neonates. Modern Care Journal, 13 (3): 1-5.

17. Platania, S., Gruttadauria, S., Citelli, G., Giambrone, L., \& Di Nuovo, S., (2017): Associations of Thalassemia Major and satisfaction with quality of life: The mediating effect of social support. Health Psychology Open, 4 (2): 1 - 9.

18. Rasbash, L., Jenkins, C., \& Laffel, L. (2016): An Integrative Review of SelfEfficacy Measurement Instruments in Youth with Type 1 Diabetes (T1DM). Diabetes Education, 41 (1): 43-58.

19. Sahmoud, S., Ibrahim, M., Toraih, E., Kamel, N., Fawzy, M., \& ElFfiky, S., (2020): Association of VDBP rs4701 Variant, but not VDR/RXR- $\alpha$ OverExpression with Bone Mineral Density in Pediatric Well-Chelated $\beta$-Thalassemia Patients. Mediterranean Journal of Hematolology and Infectious Diseases, 12 (1): 20- 3 .

20.Shareef, S., \& Obaid, K., (2015): Assessment of Knowledge of Adolescents with Thalassemia Major Regarding Iron Chelating Therapy. International Journal of Science and Research (IJSR), 6 (11): 625- 27.

21. Taheri, P., Nooryan, K., Karimi, Z., \& Zoladl, M., (2020): Effect of Individual Psychotherapy with a Focus on Self-Efficacy on Quality of Life in Patients with Thalassemia Major: A Clinical Trial. Journal of Clinical Care and Skills, 1 (2): 49- 54. 